\title{
Exoplanets search and characterization with the SOPHIE spectrograph at OHP
}

\author{
G. Hébrard ${ }^{1}$ and the SOPHIE Team \\ ${ }^{1}$ Institut d'Astrophysique de Paris, UMR7095 CNRS, Université Pierre $\mathcal{E}$ Marie \\ Curie, 98bis bd. Arago, 75014 Paris, France, $\mathcal{E}$ Observatoire de Haute-Provence, \\ 04870 Saint-Michel l'Observatoire, France [hebrard@iap.fr]
}

\begin{abstract}
Several programs of exoplanets search and characterization have been started with SOPHIE at the 1.93-m telescope of Haute-Provence Observatory, France. SOPHIE is an environmentally stabilized echelle spectrograph dedicated to high-precision radial velocity measurements. The objectives of these programs include systematic searches for exoplanets around different types of stars, characterizations of planet-host stars, studies of transiting planets through RossiterMcLaughlin effect, follow-up observations of photometric surveys. The instrument SOPHIE and a review of its latest results are presented here.
\end{abstract}

\section{Introduction}

Among the $\sim 500$ exoplanets known so far, most of them have been discovered from the reflex motion they cause to their host-star, which can be detected from stellar radial velocity wobble. Radial velocity measurements thus allow the statistics of planetary systems to be extended by completing the mass-period diagram of exoplanets, in particular towards lower masses and longer periods, as the measurement accuracy improves. In addition, radial velocity measurements are mandatory to establish the planetary nature of transiting objects detected from ground- and space-based photometric surveys. They also allow additional characterization of the detected systems, especially through the Rossiter-McLaughlin effect. Thus, radial velocity measurements remain a particularly efficient and powerful technique for research and characterization of exoplanetary systems.

High-precision radial velocity measurements have started at the 1.93-m telescope of Haute-Provence Observatory with the ELODIE spectrograph (Baranne et al. 1996). In operation from 1994 to 2006, ELODIE allowed numerous exoplanetology results to be obtained, including the detection of the first extrasolar planet 51 Peg b (Mayor \& Queloz 1995) and the famous planet transiting in front of the bright star HD 189733 (Bouchy et al. 2005), as well as the first detection of the Rossiter-McLaughlin anomaly for an exoplanetary system (Queloz et al. 2000). Among the more than twenty exoplanets that ELODIE contributed to discover, one can also quote the first planet hosted by an M dwarf (Delfosse et al. 1998), the first transiting exoplanet HD 209458b (Mazeh et al. 2000), or the extremely eccentric planet HD 80606b (Naef et al. 2001). Several instrumental effects were however restricting the ELODIE accuracy, including a low throughput, significant scattered light and limited spectral resolution. Its successor SOPHIE was developed with the main goal to improve the radial velocity accuracy.

This is an Open Access article distributed under the terms of the Creative Commons Attribution-Noncommercial License 3.0, which permits unrestricted use, distribution, and reproduction in any noncommercial medium, provided the original work is properly cited. 


\section{The SOPHIE spectrograph}

SOPHIE is a cross-dispersed, environmentally stabilized echelle spectrograph dedicated to high-precision radial velocity measurements. A detailed description of this instrument and these data is given by Perruchot et al. (2008) and Bouchy et al. (2009a). Briefly, the detector is a $4 \mathrm{k} \times 2 \mathrm{k} 15-\mu \mathrm{m}$-pixel CCD cooled at $-100^{\circ} \mathrm{C}$, with slow- and fast-readout modes. It records 39 spectral orders covering the wavelength domain from 3872 to $6943 \AA$. The charge transfer inefficiency of the detector is software-corrected (Bouchy et al. 2009b). The dispersive components are encapsulated in tank with constant pressure and temperature. The typical intrinsic drift of the spectrograph is less than $3 \mathrm{~m} / \mathrm{s}$ per hour.

The spectrograph is fed through a pair of 3"-wide optical fibers for the highresolution mode $(R=75000$, obtained from an extra slit), and another pair for the high-efficiency mode $(R=40000$, allowing one magnitude gain). The high-resolution mode is equipped with a double fiber scrambler. For each fiber pair, one aperture is used for starlight whereas the other one, 2' away, can be used either on a Thorium-Argon lamp for tracking spectrograph drift or on the sky to estimate background pollution, especially in case of strong moonlight. Both aperture can also be simultaneously put on Thorium-Argon or tungsten lamps for wavelength or flat-field calibrations, respectively.

The spectra are extracted from the detector images and the radial velocities are measured online with the SOPHIE pipeline. The spectra extraction includes localization of the spectral orders on the 2D-images, optimal order extraction, cosmic-ray rejection, wavelength calibration and spectral flat-field correction. The obtained two dimension spectra are cross-correlated with numerical masks corresponding to different spectral types and the resulting cross-correlation functions (CCFs) are fitted by Gaussians to get the radial velocities and their accuracy (Baranne et al. 1996, Pepe et al. 2002). Several stellar parameters can also be automatically estimated from the SOPHIE spectra, including the metallicity, the projected rotational velocity $v \sin i$, or the chromospheric-activity index $R_{\mathrm{HK}}^{\prime}$ (Boisse et al. 2010).

SOPHIE was mounted at the focus of the OHP 1.93-m telescope and obtained its first light in summer 2006. A few weeks after it detected its first exoplanets WASP-1b and WASP-2b (Cameron et al. 2007).

\section{Radial velocity follow-up of photometric surveys}

The importance of transiting extrasolar planets has been broadly recognized. In addition to confirm that planets detected through radial velocity variations were not artifacts due to stellar effects, they allow crucial studies to be performed. This includes planetary radius, mass, and density measurements, atmospheric absorbers detection during transits, planetary emitted light identification through occultations, accurate timing variations constraint, or obliquity estimation using the Rossiter-McLaughlin anomaly. The impact of these studies justified large efforts for the detection of transiting planets, using photometry surveys from the ground (OGLE, TrES, XO, HAT, SuperWASP) or space (CoRoT, Kepler). This results now in a biased population: over the $\sim 500$ exoplanets currently known, about 100 are transiting.

In most of cases, however, photometric data alone are not able to convincingly conclude to the detection of an extrasolar planet. Indeed, several scenarios implying binary stars could mimic the signature of a transiting planet in a light curve. This includes eclipsing $\mathrm{M}$ dwarfs as well as grazing, background or blended eclipsing binaries. 
Only a small proportion of the identified candidates actually are transiting planets. The ratio is of the order of 10 to $20 \%$ for the most efficient surveys.

Spectroscopy allows the identification of most of the scenarios implying binaries, through radial velocity measurements and line profile-shape analysis. Spectroscopic follow-up is thus mandatory to identify the actual transiting planets among the candidates detected from photometric surveys. They also allow the measurement of the mass of the secured planet and the determination of the parameters of the host star.

SOPHIE has been broadly used for the follow-up of SuperWASP candidates. This allowed the detection of the three first planets of this survey (Cameron et al. 2007, Pollacco et al. 2008), followed by a dozen of extra ones. This includes short-period planets with eccentric orbits (WASP-10b and 14b, Christian et al. 2009, Joshi et al. 2009), one of the hottest planets known (WASP-12b, Hebb et al. 2009), an inflated Saturn-mass planet (Bouchy et al. 2010a), or a giant planet on a 7-day period (WASP38b, Barros et al. 2010). In addition, the planets HAT-P-5b and HAT-P-9b have also been discovered thanks to SOPHIE follow-up (Bakos et al. 2007, Shporer et al. 2009).

CoRoT candidates have been widely followed up with SOPHIE. This allowed the detection of the first CoRoT planet (Barge et al. 2008), of other hot Jupiters including ordinary ones CoRoT-2b, 5b or 11b (Alonso et al. 2008, Rauer et al. 2009, Gandolfi et al. 2010) and some on longer periods as CoRoT-4b and 6b (Moutou et al. 2008, Fridlund et al. 2010). SOPHIE has also been used for the identification of promising candidates to be followed up at higher precision with the HARPS spectrograph. This encompasses the super Earth CoRoT-7b (Léger et al. 2009, Queloz et al. 2009) and the long-period Jupiter-mass planet CoRoT-9b (Deeg et al. 2010).

SOPHIE now is also used for the follow-up of Kepler objects of interests. This led to the confirmation of the low-mass white dwarf KOI-74b (Ehrenreich et al. 2010) and to the detection of several new transiting giant planets (Santerne et al. 2010, Bouchy et al. 2010b).

\section{Radial velocity surveys}

The SOPHIE consortium started a large and comprehensive program of radial velocity surveys to search and characterize exoplanets in the Northern hemisphere. The observing strategies and target samples are optimized to achieve a variety of science goals and to solve several important issues: 1) mass function of planets below the mass of Saturn, 2) planetary statistical properties to constrain the formation and evolution models, 3) relationships between planets and the physical and chemical properties of their stars, 4) detection of exoplanets around nearby stars, allowing space and ground-based follow-up, 5) deep characterization of known transiting exoplanets including long term follow-up and spectroscopic transit analysis. All these aspects are treated through five complementary sub-programs using an amount of 60 to 90 nights per semester (Bouchy et al. 2009b).

The first detected planets were candidates that have already been identified from former ELODIE data: HD 43691b, HD 132406b and HD 45652b (da Silva et al. 2007, Santos et al. 2008), as well as the puzzling $\theta$ Cygni case, which can be due to planets and/or stellar phenomenon. A multiple planet system was thereafter identified around HD 9446 (Hébrard et al. 2010a) as well as a giant planet around HD 109246 (Boisse et al. 2010). SOPHIE also identified companions above the $13-\mathrm{M}_{\text {Jup }}$ limit around several stars (Bouchy et al. 2009a, Díaz et al. 2010). These massive objects are at the limit between giant planets and brown dwarfs, where only a few cases are known (among 
which only three are transiting). Increasing the statistics in this massive regime is critical to understand planet formation in the high-mass limit.

A study of the stellar activity of the planet host star HD 189733 based on SOPHIE data is also presented by Boisse et al. (2008). Finally, a SOPHIE observation of a Moon eclipse allowed a simulation of the observation of the Earth seen as a transiting extrasolar planet, and the detection of several atmospheric species (Vidal-Madjar et al. 2010).

\section{Spectroscopic transits}

Thanks to the Rossiter-McLaughlin effect, spectroscopic observations of a star during the transit of its hosted planet gives informations on the obliquity of the system, i.e. the angle between the planetary orbital axis and the stellar rotation axis. This effect occurs when an object transits in front of a rotating star, causing a distortion of the stellar lines profile, and thus an apparent anomaly in the measured radial velocity of the star. The shape of the disturbed radial velocity curve allows one to determine the obliquity, or more specifically its sky-projected value $\lambda$. Such measurements could give crucial clues on the history and dynamics of planetary systems.

Between 2000 and 2008, spectroscopic transits have been measured for a dozen of extrasolar systems, including some with SOPHIE (HAT-P-2 and CoRoT-2b, Loeillet et al. 2007, Bouchy et al. 2008). All these measurements shown aligned, prograde orbits $\left(\lambda \simeq 0^{\circ}\right)$, as expected for planets that formed in a protoplanetary disk far from the star and that later migrated closer-in.

However, a first case of misaligned system was reported by Hébrard et al. (2008) in the XO-3 system. The SOPHIE observations secured during a transit of this massive planet only showed a blue-shifted anomaly, instead of the feature expected in case of aligned system. This misalignment was hereafter confirmed by Winn et al. (2009a), who however found a lower $\lambda$-value. The second case of misaligned system was also reported thanks to SOPHIE observations: simultaneously with the detection of the transiting nature of the long-period, eccentric planet HD 80606b, Moutou et al. (2009) showed that $\lambda \neq 0^{\circ}$ in this system. This result was confirmed by Pont et al. (2009) and Winn et al. (2009b), then refined thanks to the observation of another transit of this planet, simultaneously with SOPHIE and Spitzer (Hébrard et al. 2010b).

Since then, other misaligned systems were detected with SOPHIE and other spectrographs, including retrograde ones (see J. Winn and A. Triaud contributions in these proceedings). These surprising results suggest that some close-in planets might result from gravitational interaction between planets and/or stars rather than migration due to interaction with the accretion disk.

\section{Conclusion}

Established in 2006, SOPHIE is an instrument that allows significant progresses in the search and characterization of extrasolar planets. Such programs will be pursued in the years to come. On longer term, considerable efforts of radial velocity follow-up will be needed with the perspective of large space-based photometric surveys for transiting planets, like PLATO or TESS.

The present radial velocity precision obtained on stable stars with SOPHIE is about $4-5 \mathrm{~m} / \mathrm{s}$ over several semesters. This limitation is mainly due to guiding and centering effects on the fiber entrance at the telescope focal plane and the insufficient scrambling provided by the fiber and the double scrambler. An upgrade of the Cassegrain fiber 


\section{Detection and Dynamics of Transiting Exoplanets}

adapter is presently on-going, including new high-precision guiding camera and new double scrambler, with the goal to reach the precision level of 1 to $2 \mathrm{~m} / \mathrm{s}$. Such level of accuracy will allow the super-Earth domain to be explored with SOPHIE.

Acknowledgements. Most of the results presented here were obtained from current and former members of the SOPHIE Team, including L. Arnold, I. Boisse, F. Bouchy, M. Desort, X. Bonfils, X. Delfosse, R. F. Díaz, A. Eggenberger, D. Ehrenreich, T. Forveille, F. Galland, G. Hébrard, H. Le Coroller, A.-M. Lagrange, C. Lovis, M. Mayor, C. Moutou, F. Pepe, C. Perrier, F. Pont, D. Queloz, A. Santerne, N. C. Santos, D. Ségransan, S. Udry, A. Vidal-Madjar. We thank the technical team at Haute-Provence Observatory for their support with the SOPHIE instrument and the 1.93-m OHP telescope, and acknowledge fundings of the Prog. National de Planétologie (PNP) of CNRS, the Swiss National Science Foundation, and the French National Research Agency.

\section{References}

Alonso, R., Auvergne, M., Baglin, A., et al. 2008, A\&A, 482, L21

Bakos, G. A., Shporer, A., Pal, A., et al. 2007, ApJ, 671, L173

Baranne, A., Queloz, D., Mayor, M., et al. 1996, A\&AS, 119, 373

Barge, P., Baglin, A., Auvergne, M., et al. 2008, A\&A, 482, L17

Barros, S., Faedi, F., Cameron, A. et al. 2010, A\&A in press (arXiv:1010.0849)

Boisse, I., Moutou, C., Vidal-Madjar, A., et al. 2009, A\&A, 495, 959

Boisse, I., Eggenberger, A., et al. 2010, A\&A, in press (arXiv:1006.4984)

Bouchy, F., Udry, S., Mayor, M., et al. 2005, A\&A, 444, L15

Bouchy, F., Queloz, D., Deleuil, M., et al. 2008, A\&A, 482, L2

Bouchy, F., Hébrard, G., Udry, S., et al. 2009a, A\&A, 505, 853

Bouchy, F., et al. 2009b, EAS Publications Series, 37, 247

Bouchy, F., Hebb, L., Skillen, I., et al. 2010a, A\&A, 519, 98

Bouchy, F., et al., 2010, in preparation

Cameron, A., Bouchy, F., Hébrard, G., et al. 2007, MNRAS, 375, 951

Christian, D., Gibson, N., Simpson, E., et al. 2009, MNRAS, 392, 1585

da Silva, R., Udry, S., Bouchy, F., et al. 2007, A\&A, 473, 323

Deeg, H. J., Moutou, C., Erikson, A., et al. 2010, Nature , 464, 384

Delfosse, X., Forveille, T., Mayor, M., et al. 1998, A\&A, 338, L67

Díaz, R. F., et al. 2010, in preparation

Ehrenreich, D., Lagrange, A.-M., et al. 2010, A\&A, in press (arXiv:1009.1873)

Fridlund, M., Hébrard, G., Alonso, R., et al. 2010, A\&A, 512, 14

Gandolfi, D., Hébrard, G., et al. 2010, A\&A, in press (arXiv:1009.2597)

Hebb, L., Collier-Cameron, A., Loeillet, B., et al. 2009, ApJ, 693, 1920

Hébrard, G., Bouchy, F., Pont, F., et al. 2008, A\&A, 488, 763

Hébrard, G., Bonfils, X., Ségransan, D., et al. 2010a, A\&A, 513, 69

Hébrard, G., Désert, J.-M., Díaz, R. F., et al. 2010b, A\&A, 516, 95

Joshi, Y. C., Pollacco, D., Cameron, A., et al., 2009, MNRAS, 392, 1532

Léger, A., Rouan, D., Schneider, J., et al. 2009, A\&A, 506, 287

Loeillet, B., Shporer, A., Bouchy, F., et al. 2007, A\&A, 481, 381

Mayor, M., \& Queloz, D. 1995, Nature , 378, 355

Mazeh, T., Naef, D., Torres, G., et al. 2000, ApJ, 532, L55

Moutou, C., Bruntt, H., Guillot, T., et al. 2008, A\&A, 488, L47

Naef, D., Latham, D. W., Mayor, M., et al. 2001, A\&A, 375, L27 


\section{EPJ Web of Conferences}

Pepe, F., Mayor, M., Galland, F., et al. 2002, A\&A, 388, 632

Perruchot, S., Kohler, D., Bouchy, F., et al., 2008, SPIE, 70140J

Pollacco, D., Skillen, I., Collier Cameron, A., et al. 2008, MNRAS, 385, 1576

Pont, F., Hébrard, G., Irwin, J. M., et al. 2009, A\&A, 509, 695

Queloz, D., Eggenberger, A., Mayor, M., et al. 2000, A\&A, 359, L13

Queloz, D., Bouchy, F., Moutou, C., et al. 2009, A\&A, 506, 303

Rauer, H., Queloz, D., Csizmadia, S., et al. 2009, A\&A, 506, 281

Santerne, A., Díaz, R. F., Bouchy, F., et al. 2010, submitted to A\&A

Santos, N. C., Udry, S., Bouchy, F., et al. 2008, A\&A, 487, 369

Shporer, A., Bakos, G., Bouchy, F., et al. 2009, ApJ, 690, 1393

Vidal-Madjar, A., Arnold, L., et al. 2010, A\&A, in press (arXiv:1007.0008)

Winn, J. N., Johnson, J. A., Fabrycky, D., et al. 2009, ApJ, 700, 302

Winn, J. N., Howard, A. W., Johnson, J. A., et al. 2009b, ApJ, 703, 2091 